\title{
Chlamydophila pneumoniae Infection Leads to Smooth Muscle Cell Proliferation and Thickening in the Coronary Artery without Contributions from a Host Immune Response
}

\author{
Justin F. Deniset, Paul K.M. Cheung, \\ Elena Dibrov, Kaitlin Lee, Sarah Steigerwald, \\ and Grant N. Pierce \\ From the Institute of Cardiovascular Sciences, St. Boniface \\ General Hospital Research Centre, Department of Physiology, \\ Faculties of Medicine and Pharmacy, University of Manitoba, \\ Winnipeg, Manitoba, Canada
}

Cblamydophila pneumonia (C. pneumonia) infection has been associated with the progression of atherosclerosis. It remains unclear, however, whether $C$. pneumoniae in the absence of an immune response can alone initiate atherogenic events within a complex vessel environment. Left anterior descending coronary arteries isolated from porcine hearts were dissected and placed in culture medium for 72 hours before infection with $C$. pneumoniae. $C$. pneumoniae replicated within the arterial wall for the duration of the experiment (up to 10 days). A significant increase in chlamydial-HSP60 protein expression from day 2 to 10 post-infection (pi) indicated the presence of metabolically active $C$. pneumonia within infected vessels. Significant arterial thickening in infected coronary segments was observed by a considerable decrease in the ratio of lumen to total vessel area (48 \pm $3 \%$ at day 4 pi versus $23 \pm 3 \%$ at day 10 pi) and a significant increase in the ratio of media to luminal area $(113 \pm 16 \%$ at day 4 pi versus $365 \pm 65 \%$ at day 10 pi). Structural changes were accompanied by an up-regulation of host HSP60 and proliferating cell nuclear antigen expression levels. Immunohistochemical staining confirmed proliferating cell nuclear antigen expression to be primarily localized within smooth muscle cells of the medial area. These results demonstrate that $C$. pneumoniae infection can stimulate arterial thickening in a complex vessel environment without the presence of a host immune response and further supports the involvement of
HSP60 in this action. (Am J Pathol 2010, 176:1028-1037; DOI: 10.2353/ajpath.2010.090645)

Chlamydophila pneumoniae (C. pneumoniae) is an obligate intracellular parasite that causes respiratory illness in humans. It is a widespread pathogen, and up to $50 \%$ of adults by the age of 20 may carry serological evidence of exposure. ${ }^{1}$ In the cytoplasm of an infected cell, C. pneumoniae multiplies within membrane bound vacuoles termed as the chlamydial inclusions or reticulate body. Active metabolism occurs in the reticulate body which imports ATP, amino acids, nucleoside (RNA) precursors, and other essential molecules from the host cytoplasm. ${ }^{2-4}$ At 48 to 72 hours post infection (pi), the reticulate bodies differentiate into infectious progeny particles, elementary bodies. Currently, the molecular mechanisms involved at the various stages of the $C$. pneumoniae life cycle remain unclear.

The clinical significance of $C$. pneumoniae goes beyond respiratory illness. Several in vitro investigations have suggested that $C$. pneumoniae, after infecting monocytes of the respiratory tract, travels through the circulatory system and disseminates at the endothelial surface of susceptible arterial lesions. ${ }^{5-7}$ The first association of $C$. pneumoniae infection with coronary artery disease (CAD) was reported by Saikku et al in

\footnotetext{
Supported by a grant from the Canadian Institutes for Health Research and from the St. Boniface Hospital and Research Foundation. J.D. received a Frederick Banting and Charles Best Canada Graduate Scholarship from CIHR. P.C. received a postdoctoral fellowship from the Focus on Stroke Initiative of CIHR/HSFC/Canadian Stroke Network/ AstraZeneca Canada and the CIHR IMPACT strategic Post-Doctoral Training Program.

Accepted for publication October 20, 2009.

Address reprint requests to Dr. Grant N. Pierce, Institute of Cardiovascular Sciences, St. Boniface General Hospital Research Centre, 351 Tache Avenue, Winnipeg, Manitoba, Canada R2H 2A6. E-mail: gpierce@ sbrc.ca.
} 
1988. ${ }^{8}$ Since then, numerous studies have confirmed a strong correlation of $C$. pneumoniae infection with coronary arterial diseases. ${ }^{5,6,9-12}$ Furthermore, live $C$. pneumoniae and C. pneumoniae-specific T-cells were detected in coronary and carotid atherosclerotic plaque, ${ }^{13,14}$ but not in neighboring healthy tissues. ${ }^{15-17}$ The use of antibiotics in patients with CAD has been shown to be beneficial. ${ }^{18-20}$

The mechanism whereby $C$. pneumoniae infection stimulates CAD is unclear. However, C. pneumoniae infection was shown to induce atherogenesis in vivo in an hypercholesterolemic animal model. ${ }^{21} \mathrm{C}$. pneumoniae infection stimulates foam cell formation in atheroma, ${ }^{22-24}$ and induces proliferation of vascular smooth muscle cells (VSMCs). ${ }^{25-27}$ Furthermore, C. pneumoniae infection leads to up-regulation of endogenous heat-shock protein 60 (HSP60), ${ }^{27}$ shown to be a major contributor of an autoimmune response during the development of atherosclerosis. ${ }^{28-30}$ Overexpression of endogenous HSP60 directly induced VSMC proliferation, ${ }^{27}$ an event identified as an important component of the atherogenic process.

The contribution of the immune response to the proliferative action of the infectious stimulant has been hypothesized to be an important component of the atherogenic response. ${ }^{31}$ Many other infectious agents have also been shown to stimulate atherosclerosis, ${ }^{32,33}$ suggesting a common immune/inflammatory response pathway in the atherogenic action. The study of $C$. pneumoniae-induced atherogenesis on any in vivo model is inevitably complicated by the contributions of host-immune and inflammatory responses (T-cells and cytokines), which are thought to be strong contributors to the lesions. C. pneumoniae infection can augment the secretion of inflammatory markers by endothelial cells. ${ }^{25-27}$ The up-regulation of HSP60 in human atheroma and the corresponding elevated levels HSP60 antibodies can also lead to autoimmune injury in the arterial environment. ${ }^{29,34-37}$ However, the question of whether C. pneumoniae infection directly injures coronary arteries and induces thickening without the effects of the immune system remains unanswered. It is difficult to answer this question in an in vivo environment because the immune system is fully activated by the infectious agent. Data from in vitro studies are not ideal for studying C. pneumoniae induced proliferation and thickening because cell lines are naturally proliferative and lack the structural complexity of a vessel. In the current study, we used a novel ex vivo organ culture model that allowed us to study the direct consequences of the $C$. pneumoniae infection in the complex arterial environment without the confounding contributions of a host immune system. Using this novel approach, we observed C. pneumoniae replication and the spread of the infection into the medial layer of coronary arteries. Furthermore, $C$. pneumoniae infection induced arterial wall thickening and expression of proliferation and stress markers. This is the first report that shows that $C$. pneumoniae infection can stimulate an atherogenic response independently of the host immune reactions.

\section{Materials and Methods}

\section{Reagents and Antibodies}

Cycloheximide, bradykinin acetate, 9,11-Dideoxy-11 $\alpha$, $9 \alpha$-epoxy-methanoprostaglandin $F_{2 \alpha}$ (u46619), sodium nitroprusside, Hoescht, anti-proliferating cell nuclear antigen (PCNA) antibody, and anti-smooth muscle actin antibody were obtained from Sigma-Aldrich (St-Louis, $\mathrm{MO}$ ). The anti-Chlamydia genus antibody was obtained from Argene (Verniolle, France). The anti-PCNA antibody was obtained from Bethyl Laboratories Inc. (Montgomery, TX). The anti-chlamydial heat-shock protein 60 antibody was obtained from Affinity BioReagents (Golden, CO). The anti-mammalian heat-shock protein 60 was obtained from Assay Designs-Stressgen (Ann Arbor, MI). The glyceraldehyde-3-phosphate dehydrogenase (GAPDH) antibody was obtained from Abcam (Cambridge, MA). The HRP conjugated anti-mouse IgG was obtained from Millipore (Billerica, MA). The Alexa-488-conjugated antimouse IgG and Alexa-488-conjugated anti-rabbit IgG antibodies were obtained from Invitrogen (Carlsbad, CA). The Texas Red-conjugated anti-mouse IgG antibody was obtained from Jackson Laboratory (Bar Harbor, ME).

\section{Propagation of $\mathrm{C}$. pneumoniae}

C. pneumoniae AR39 strain was obtained from the University of Washington, Seattle, WA. The organism was propagated in HL cells as described previously. ${ }^{38}$ The purified organism was resuspended in chlamydial sucrosephosphate-glutamate medium and stored at $-80^{\circ} \mathrm{C}$ until use. The titer of C. pneumoniae was determined in cycloheximide-treated $\mathrm{HL}$ cells, and concentrations used were expressed as inclusion-forming units per $\mathrm{ml}^{39}$

\section{Porcine Coronary Explant and Organ Culture}

The coronary artery explant and organ culture method has been adapted from Saward et al. ${ }^{40}$ Whole hearts from 10-month-old neutered male swine were obtained from a local abattoir. The left anterior descending coronary artery was flushed with standard phosphate saline buffer (PBS) supplemented with an antibiotic mixture (penicillin G ,1500 units/ml; streptomycin sulfate, 1500 $\mu \mathrm{g} / \mathrm{ml}$; amphotericin B, $2.5 \mu \mathrm{g} / \mathrm{ml}$; Invitrogen) and subsequently dissected out of the heart and cleaned of adhering fat and connective tissue. The explanted artery was cut into segments of $5 \mathrm{~mm}$ in length and incubated at $37^{\circ} \mathrm{C}$ and $5 \% \mathrm{CO}_{2}$ in organ culture medium (Dulbecco's Modified Eagle's Medium; Invitrogen, supplemented with $20 \%$ fetal bovine serum; Hyclone) supplemented with antibiotics (penicillin G ,1050 units/ml; streptomycin sulfate, $1050 \mu \mathrm{g} / \mathrm{ml}$; amphotericin B, $2.5 \mu \mathrm{g} / \mathrm{ml}$; Invitrogen). Every 24 hours, explants were placed in fresh organ culture medium with gradually decreasing amount of antibiotics. At 72 hours, the coronary segments were washed with PBS and incubated in the organ culture medium for 3 hours. The segments were then infected 
with C. pneumoniae. No antibiotics were used for the duration of the experiment.

\section{Infection of Coronary Segments with C. pneumoniae}

To perform the infection, each coronary segment was placed into an isolated well of a 96-well culture plate. The segments were oriented upright within the well so that $C$. pneumoniae $\left(5 \times 10^{6}\right.$ inclusion-forming units in $\left.100 \mu \mathrm{l}\right)$ could be applied directly into the lumen of each coronary vessel. After three hours of incubation at $37^{\circ} \mathrm{C}$ and $5 \%$ $\mathrm{CO}_{2}$, each coronary segment was transferred to an isolated well of a 24-well plate containing $1.5 \mathrm{ml}$ of organ culture medium. The vessels were incubated for a maximum of 10 days. The incubation medium was changed every 48 hours. Coronary segments were collected for analysis immediately after infection and from day 2 to day 10 pi. Heat-inactivated C. pneumoniae (mock-infection) was used for controls in each experiment.

\section{Immunofluorescent Microscopy}

Infected and mock-infected coronary segments were collected at various time points pi: day 2 , day 4 , day 6 , day 8, and day 10. The segments were fixed in ethanol, rehydrated, and subsequently equilibrated in 30\% sucrose solution before mounting in OCT compound (Tissuetek). These OCT-mounted segments were slowly frozen to $-80^{\circ} \mathrm{C}$, cut (HM 500 OM Cryostat, Microm) into 6 - $\mu \mathrm{m}$ sections, and placed on positively coated glass slides (Fisher). OCT was washed off the glass slides with PBS, and antigen retrieval was performed with the Unmasking solution (Vector Laboratories) at $65^{\circ} \mathrm{C}$ for 25 minutes. To detect $C$. pneumoniae in the specimen, anti-Chlamydia genus antibody (1:100) was applied according to manufacturer's protocol. Alexa-488-conjugated anti-mouse IgG (1:800) was used as a secondary antibody. To localize PCNA expression within the vessel, anti-smooth muscle actin (1:500) and anti-PCNA (1:100) were used. Texas Red-conjugated anti-mouse IgG (1:1000) and Alexa-488-conjugated anti-rabbit IgG (1:1000) were used as secondary antibodies for the respective primary antibodies. Hoescht staining solution (5 ng/ml) was added to the slides to identify nuclei. Slides were then fixed with FluoroSave reagent (Calbiochem) to preserve fluorescence. The location and number of $C$. pneumoniae inclusion bodies (IB) in the coronary cross-sections were visualized as green fluorescent signals at 40X magnification on an inverted microscope (Nikon, TE-2000s). In each field of view, the number of fluorescent IBs was calculated using imaging software (Adobe photoshop CS3 extended). To identify apoptotic cells within the vessel, terminal dUTP nick-end labeling (TUNEL) assays using the FragEL DNA fragmentation detection kit (Calbiochem) were performed on coronary cross-sections. Cross-sections pretreated with DNase I were used as positive controls.

\section{Histological Staining}

To determine the extent of media thickening, coronary segments were collected at four time points pi: day 4, day 6 , day 8 , and day 10 . The extent of media thickening in $C$. pneumoniae-infected segments was compared with corresponding mock-infected control segments. The segments were fixed and mounted as described above before being cut into $6-\mu \mathrm{m}$ cross-sections. Hematoxylin and eosin $(\mathrm{H} / \mathrm{E})$ staining as well as elastic staining were applied to these cross-sections. Images of the cross-sections were obtained at $4 \times$ magnification (Nikon TE-2000s). Imaging software (Adobe photoshop CS3 extended) was used to quantify the pixel area of the lumen, media, and total vessel for each coronary cross section.

\section{Western Blot Analysis}

To assess protein expression levels, coronary segments were collected at four time points: day 0 (before infection), days 2 to 4 , days 5 to 6 , and days 8 to 10 pi. These collection intervals were chosen to coincide with the $C$. pneumoniae replication cycle. Three independent experiments were performed. In each experiment, two coronary segments per time point per treatment were collected, rinsed with PBS, flash-frozen in liquid nitrogen and subsequently stored at $-80^{\circ} \mathrm{C}$. Paired coronary segments were ground and resuspended in RIPA buffer $(50 \mathrm{mmol} / \mathrm{L}$ TrisHCl $\mathrm{pH} 7.5,150 \mathrm{mmol} / \mathrm{L} \mathrm{NaCl}, 1 \mathrm{mmol} / \mathrm{L}$ EDTA, $1 \mathrm{mmol} / \mathrm{L}$ EGTA, $1 \%$ Triton 100, 0.1\% SDS, 0.5\% Na Deoxycholate, $1 \mu \mathrm{g} / \mathrm{ml}$ Leupeptin, $1 \mathrm{mmol} / \mathrm{L}$ PMSF, $1 \mathrm{mmol} / \mathrm{L}$ protease inhibitor cocktail, $1 \mathrm{mmol} / \mathrm{L}$ DTT and $1 \mathrm{mmol} / \mathrm{L}$ Benzimidine). Pro-
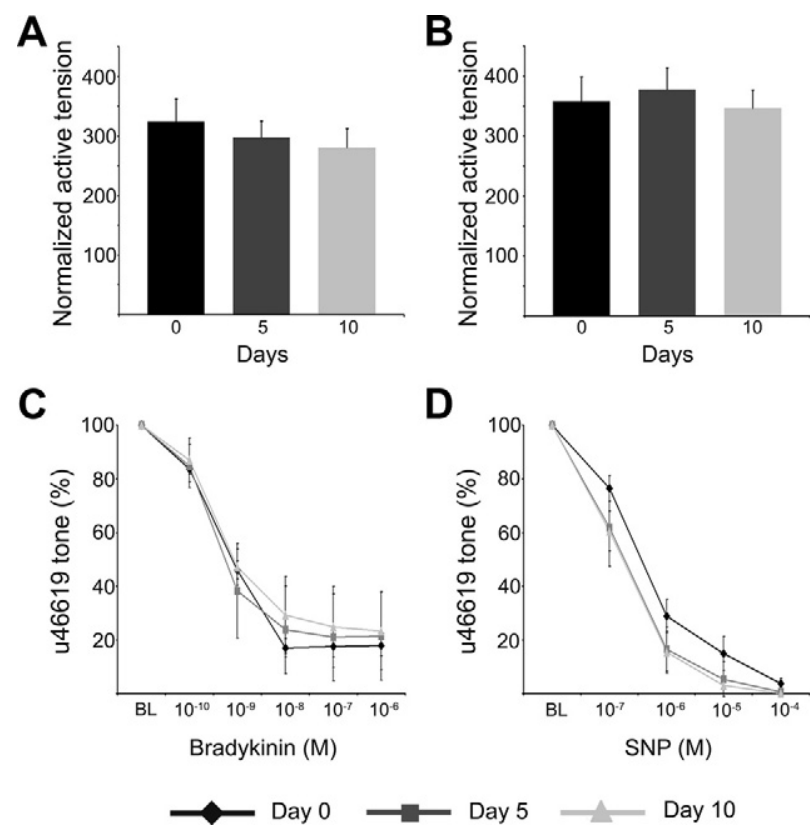

D

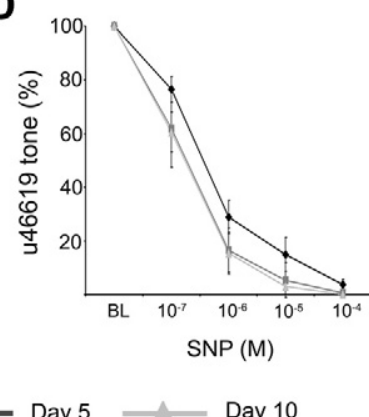

Figure 1. Contractile response of isolated vascular rings from pig coronary arteries as a function of time in organ culture medium. Maximal contractile response to $47 \mathrm{mmol} / \mathrm{L} \mathrm{KCl}(\mathbf{A})$ or $300 \mathrm{nmol} / \mathrm{L} \mathrm{u} 46619$ (B). Relaxation of rings to $10^{-10}$ to $10^{-6}$ bradykinin $(\mathbf{C})$ or $10^{-7}$ to $10^{-4}$ sodium nitroprusside (D) after precontraction with $30 \mathrm{nmol} / \mathrm{L}$ u 46619. All values were the mean \pm SEM for $n=4$ to $6 ; P>0.05$ vs. 0 time point. 


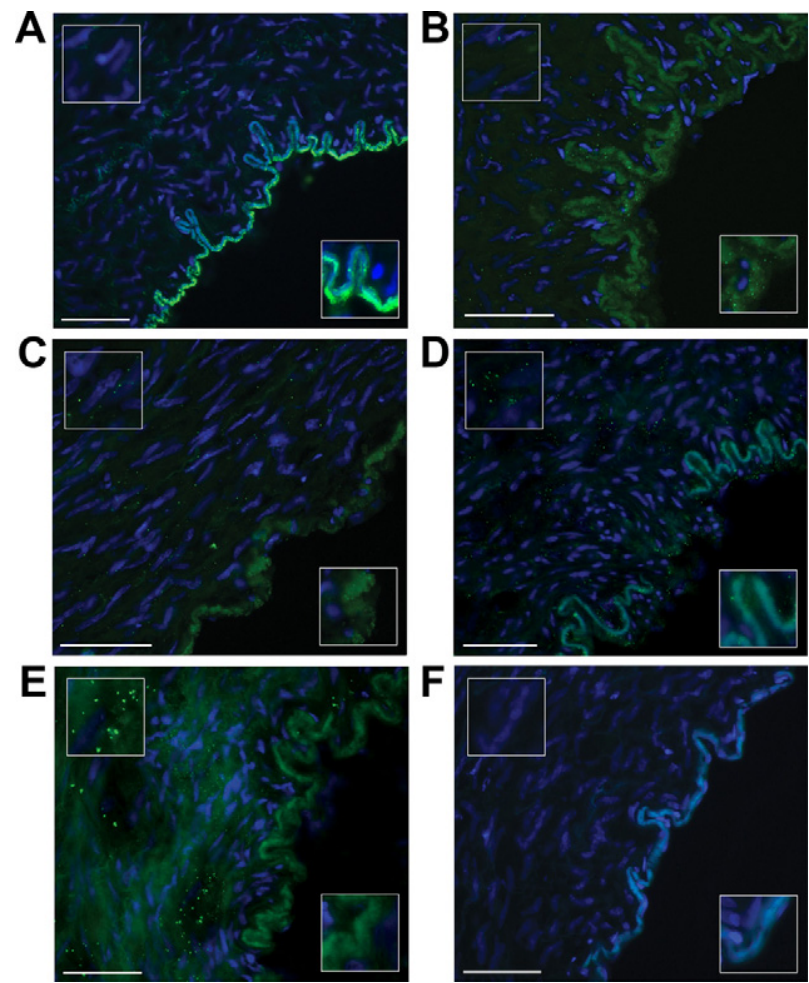

Figure 2. Immunofluorescent detection of $C$ pneumoniae inclusion bodies within infected coronary rings. A: Day 2 pi; B: Day 4 pi; C: Day 6 pi; D: Day 8 pi; E: Day 10 pi; F: Mock infection. C. pneumoniae MOMP is represented as fluorescent green signals, and nuclear staining is observed as blue. Magnified images of both the medial layer and endothelium are found in the top left and bottom right corners of each panel, respectively. Scale bar $=50 \mu \mathrm{m}$

teins were separated using a $10 \%$ denaturing polyacrylamide gel and transferred electrophoretically onto a nitrocellulose membrane. Membranes were incubated with anti-chlamydial heat-shock protein 60, anti-mammalian heat-shock protein 60, and anti-PCNA antibodies. HRP conjugated anti-mouse IgG was used as a secondary antibody. The level of GAPDH was used as a loading control. Bands were visualized with the Supersignal West Pico Chemiluminescent Substrate
(Pierce) and subsequently quantified by densitometry (Software: Quantity One, Bio-Rad).

\section{Vascular Function Assessment}

To determine tissue viability over the duration of the experiment, untreated coronary segments were collected at days 0,5 , and 10 pi to coincide with the start, mid-point, and end of the infection timeline. These tissues were used for vascular function experiments. The 5-mm coronary rings were fastened in an organ bath with surgical wire, perfused with Krebs-Henseleit solution, aerated with $95 \% \mathrm{O}_{2}$ and $5 \% \mathrm{CO}_{2}$, and equilibrated at $37 \mathrm{C}$ and $\mathrm{pH}$ 7.4. Vascular function was measured with a force transducer (FSG-01/20, Experimentia Ltd, Budapest, Hungary) as mechanograms of tension. The coronary segments were brought to a basal tension of $5 \mathrm{~g}$ and then contracted three times with $47 \mathrm{mmol} / \mathrm{L} \mathrm{KCl}$, with washout periods using Krebs solution between each contraction. To assess relaxation responses, rings were precontracted with $30 \mathrm{nmol} / \mathrm{L}$ u46619 and allowed to reach a steady state of contraction. Bradykinin was then administered without washout at concentrations of $10^{-10}$ to $10^{-6} \mathrm{~mol} / \mathrm{L}$ to develop an endothelial-dependent response curve. After washout and a second precontraction with $30 \mathrm{nmol} / \mathrm{L}$ u46619, sodium nitroprusside was administered at concentrations of $10^{-7}$ to $10^{-4}$ to develop an endothelial independent response curve. After a washout, a dose-response curve to u46619 was constructed with concentrations of 0.3 to $300 \mathrm{nmol} / \mathrm{L}$.

\section{Statistical Analysis}

Data are presented as mean \pm SEM unless otherwise stated. Differences in C. pneumoniae infected and mock C. pneumoniae infected data including the levels of protein expression, the ratio of lumen area to vessel area, the ratio of media to lumen, and the number of inclusion bodies in coronary cross-sections were tested by one-way analysis of variance followed by a Duncan's post hoc test. A probability of $P<0.05$ was considered statistically significant.
A

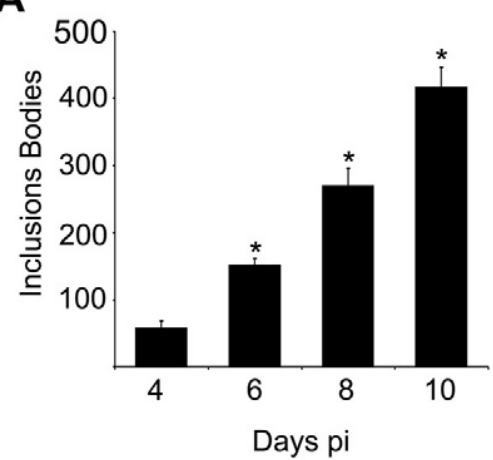

B

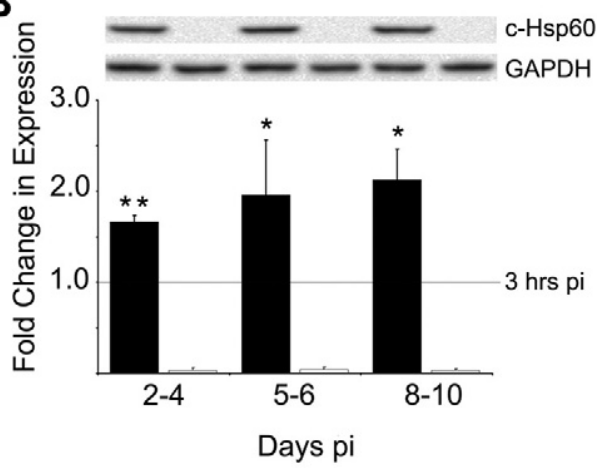

Figure 3. C. pneumoniae replication and metabolic activity within isolated coronary sections. A: The number of $C$. pneumoniae inclusion bodies at days 4 to $10 \mathrm{pi}$ (mean \pm SEM, $n=3,{ }^{*} P<$ 0.001). B: Western blot analysis of chlamydial HSP60 (c-HSP60) expression in the coronary segments over the course of infection. Representative Western blots of c-HSP60 and GAPDH (loading control) are displayed above the graph Normalized protein expression levels were represented as fold increase of the basal level. Basal level of c-HSP60 was assessed by vessels infected by the bacteria for 3 hours. ( ${ }^{*} P<0.05$ versus basal level. ${ }^{* *} P<0.01$ versus mockinfected control, $n=3$ ). 


\section{Results}

\section{Isolated Coronary Segments Maintain} Functional Viability in Culture

Vascular function was determined in untreated coronary vessels at days 0,5 , and 10 pi. There were no significant changes in maximal contractile force development to $\mathrm{KCl}$ or to $\mathrm{u} 46619$ at any of the day points (Figure 1, A and B). Furthermore, both endothelial-dependent and -independent relaxation response curves to bradykinin and so- dium nitroprusside, respectively, were maintained for the duration of the experiment (Figure 1, C and D).

\section{C. pneumoniae Propagates in the Coronary Arterial Environment}

Chlamydial IBs are represented as green fluorescent signals within the coronary cross-sections (Figure 2). Magnified views of both the endothelial layer (bottom right corner) and the medial layer (top left corner) are
A

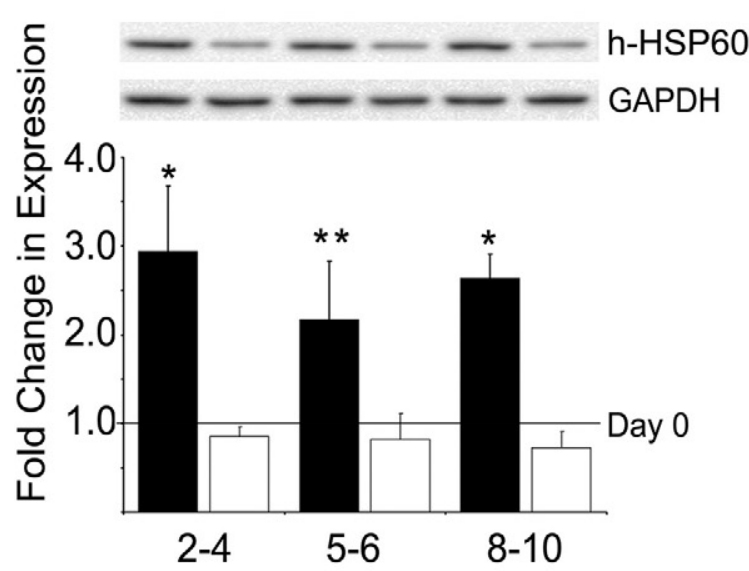

Days pi

B

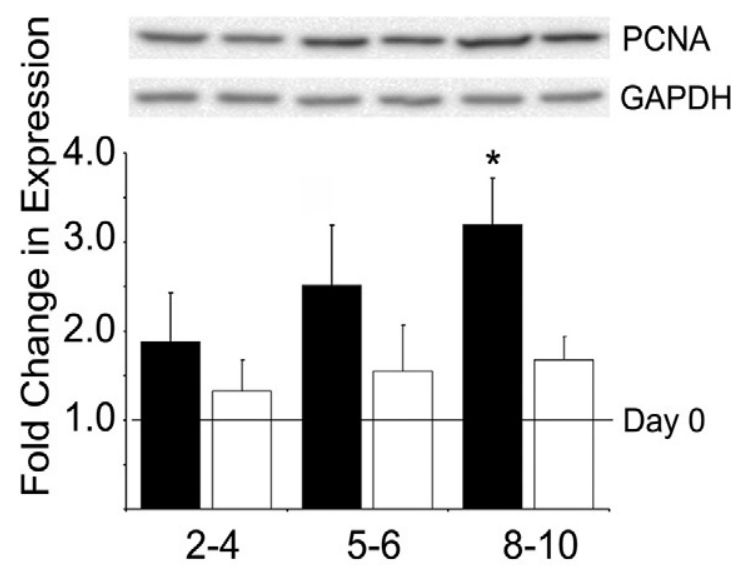

Days pi
C
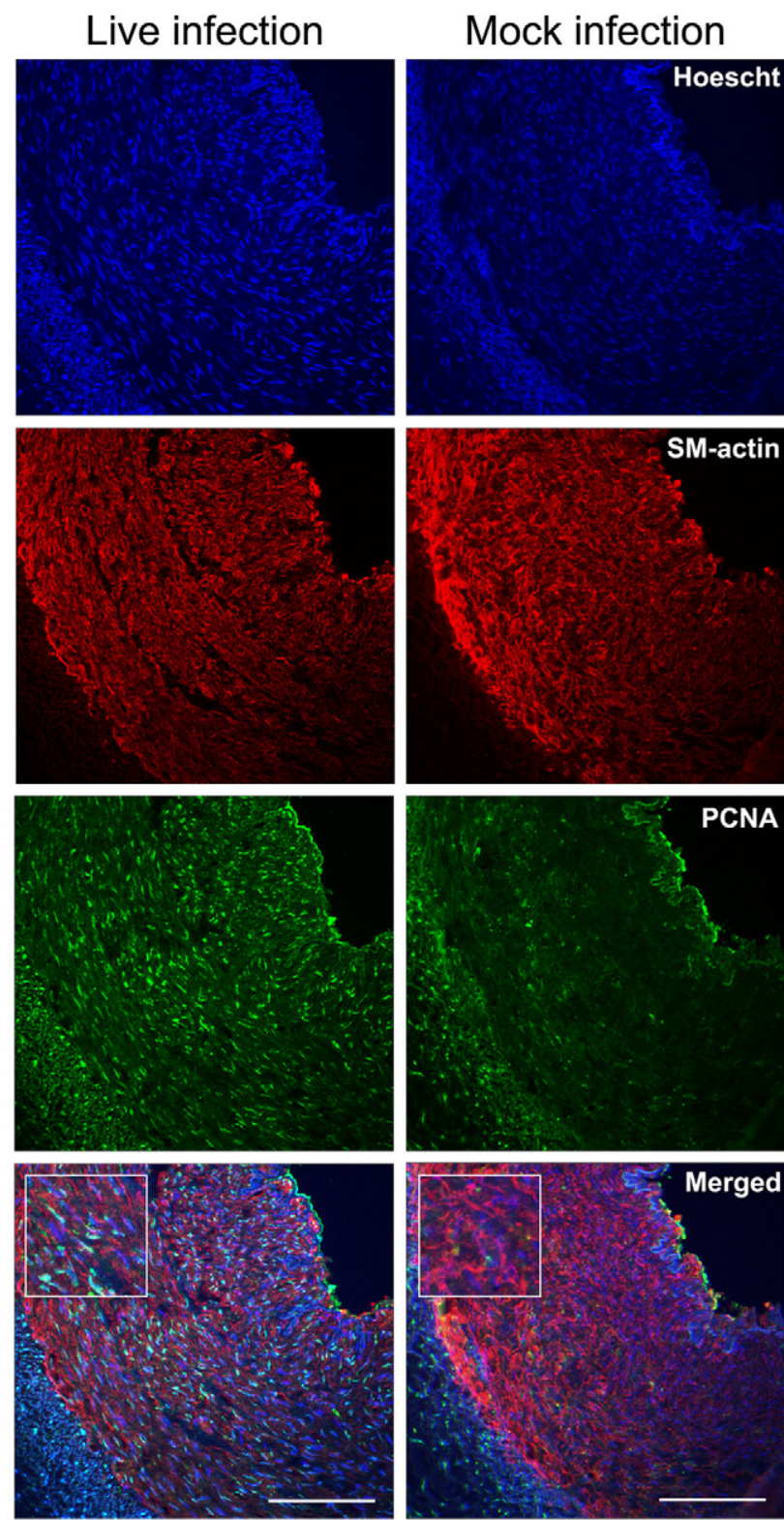

Figure 4. Western blot analysis of host-HSP60 (h-HSP60) (A) and PCNA expression (B) in coronary segments over the course of infection. Representative Western blots for m-HSP60 and PCNA along with GAPDH are displayed above their corresponding graphs. Normalized protein expression levels were represented as fold increase of the basal level (Day 0, vessels collected just before infection). ${ }^{*} P<0.05$ versus basal level and mock infected control; ${ }^{* *} P<0.05$ versus mock-infected control, $n=3$ ). C: Immunohistochemistry staining of vessels collected at day 10 pi. Left column, mock infection; right column, live (Cpn) infection. Hoescht, blue; $\alpha$-smooth muscle actin, red; $\alpha$-PCNA, green. Scale bar $=200 \mu \mathrm{m}$. 
provided within each figure panel for improved visualization of inclusion body distribution. C. pneumonia infected tissues displayed the presence of IBs at all time points, with variations in their distribution, number, and intensity. On day 2 pi, intense green fluorescence was localized in the endothelial layer but not in the smooth muscle cells (Figure 2A). On day-4 pi, C. pneumoniae IBs were still detected in the endothelium but were now also visible in the smooth muscle layer (Figure 2B). This distribution trend continued through days 6,8 , and $10 \mathrm{pi}$. At these time points, IBs were primarily localized in the media and only occasionally in the endothelium (Figures 2, C, D, E, and $F$ ). The number of IBs were quantified (Figure 3A), with the exception of IBs at day-2 pi, when it was too difficult to resolve the continuum of green fluorescence in the endothelial layer into individual IB units. From days 4 to $10 \mathrm{pi}$, a significantly $(P<0.0003)$ increasing number of IB was observed. C. pneumoniae IBs at days 8 and 10 pi were also noticeably larger in size than IBs detected at earlier day points. There were no C. pneumoniae IBs detected in mock-infected controls.

To further confirm the metabolic activity of the C. pneumoniae infection, chlamydial HSP60 expression was quantified by Western blot analysis (Figure 3B). From days 4 to $10 \mathrm{pi}$, expression of chlamydial HSP60 significantly increased from $1.67 \pm 0.07$ to $2.13 \pm 0.33$-fold $(P<0.01)$ over baseline values (the amount of chlamydial HSP60 detected at 3 hours). Chlamydial HSP60 was not observed in mock-infected control. These results demonstrate that $C$. pneumoniae was able to infect isolated coronary arteries and actively replicate within the vessel over a period of 10 days in culture.

\section{C. pneumoniae Infection Stimulates Mammalian HSP60 and PCNA Expression}

Previous work from our lab has shown that host HSP60 expression (h-HSP60) was induced during the C. pneumoniae infection, and this stimulated SMC proliferation. PCNA was used as a reliable marker of cell proliferation. ${ }^{41}$ Expression levels of h-HSP6O and PCNA from infected and mock-infected coronary segments were determined by Western blot analysis (Figure 4). Live C. pneumoniae infection strongly induced expression of hHSP60 (2.94 $\pm 0.74[P<0.005], 2.17 \pm 0.65$, and $2.64 \pm$

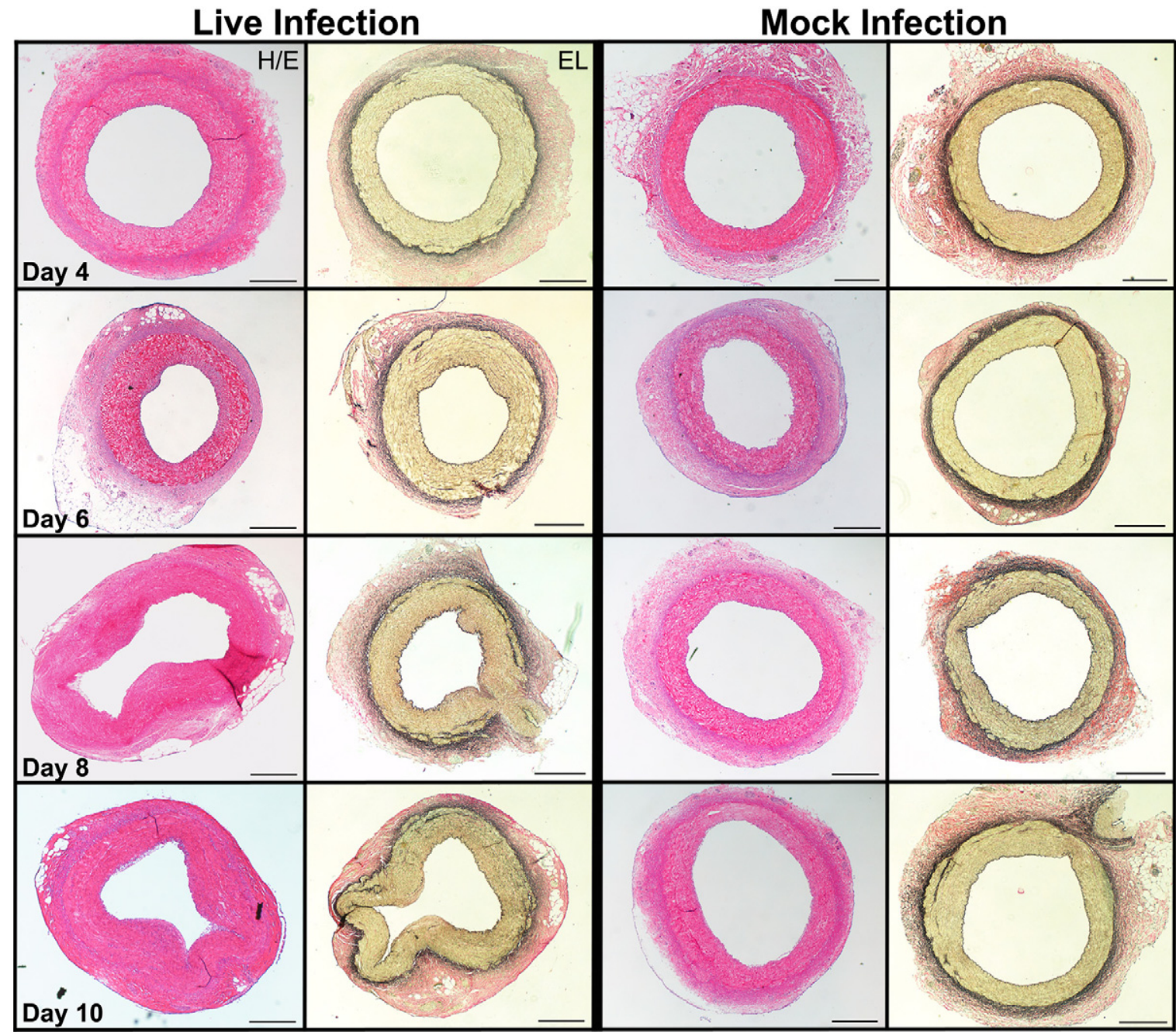

Figure 5. Representative hematoxylin/eosin ( $\mathrm{H} / \mathrm{E}$, left panel) and elastic (EL, right panel) of the infected coronary segments at day 4 , 6 , 8 , and 10 pi. Left columns, live $(\mathrm{Cpn})$ infection; right columns, mock infection. Scale bar $=500 \mu \mathrm{m}$. 
$0.27[P<0.005]$ fold over basal levels [day 0$]$ at days 3 to 4,5 to 6 , and 8 to 10 pi, respectively). These increases were all statistically significant when compared with hHSP60 expression levels in mock-infected coronary segments, all of which remained at basal levels (day 0; Figure 4A). Similarly, expression levels of PCNA in infected coronary segments gradually increased throughout all three time points, up to a maximum fold increase of $3.20 \pm 0.52(P<0.005)$ over baseline on days 8 to $10 \mathrm{pi}$ (Figure 4B) This increase was statistically significant compared with expression levels in mock-infected vessels. Mock-infected segments did not exhibit a statistically significant increase in PCNA expression over the baseline. The expression pattern of PCNA within the vessel wall was assessed in infected vessels collected at day 10 pi via immunohistochemistry techniques (Figure 4C). A marked difference in PCNA expression between treatments was again observed. Furthermore, PCNA expression in the $C$. pneumoniae-infected vessels was primarily localized within the nucleus of smooth muscle cells in the medial layer.

We have previously shown that C. pneumoniae infection in culture induces cell death in endothelial cells. However, there was no evidence of apoptosis in the present conditions as detected by TUNEL labeling as a function of C. pneumoniae infection (data not shown).

\section{C. pneumoniae Infection of Explanted Vessels Induces Medial Thickening}

The PCNA data above would suggest that smooth muscle cells are entering the cell cycle in the C. pneumoniaeinfected vessels, but it is not clear whether this process is continuing to termination. Tissue thickening would provide clear evidence of this response. Representative $\mathrm{H} / \mathrm{E}$ and elastic staining at days $4,6,8$, and 10 pi of both $C$. pneumoniae and mock-infected coronary segments are shown in Figure 5. The extent of media thickening was determined by the calculation of two separate ratios from specific arterial areas. The ratio of luminal area to total vessel area (Figure 6A) represents the degree of arterial thickening, whereas the ratio of medial area to luminal area (Figure 6B) shows the primary site for the thickening. The C. pneumoniae-infected segments displayed a progressive regression in lumen to total vessel area: $48 \pm$ $3 \%$ (day 4 pi), $36 \pm 2 \%$ (day 6 pi), $26 \pm 2 \%$ (day 8 pi), and $23 \pm 3 \%$ (day 10 pi). Furthermore, the ratio of media to luminal area was significantly increased from $113 \pm$ $16 \%$ on day 4 pi to a maximum of $365 \pm 66 \%$ on day 10 pi $(P<0.02)$. In contrast, ratios for mock-infected tissues remained unchanged from days 4 to 10 pi (Figure 6, A and $B$ ). These data demonstrate that $C$. pneumoniae infection has the ability to induce media thickening in isolated coronary arteries.

\section{Discussion}

Strong clinical correlations between C. pneumoniae infection and coronary artery disease have been repor-
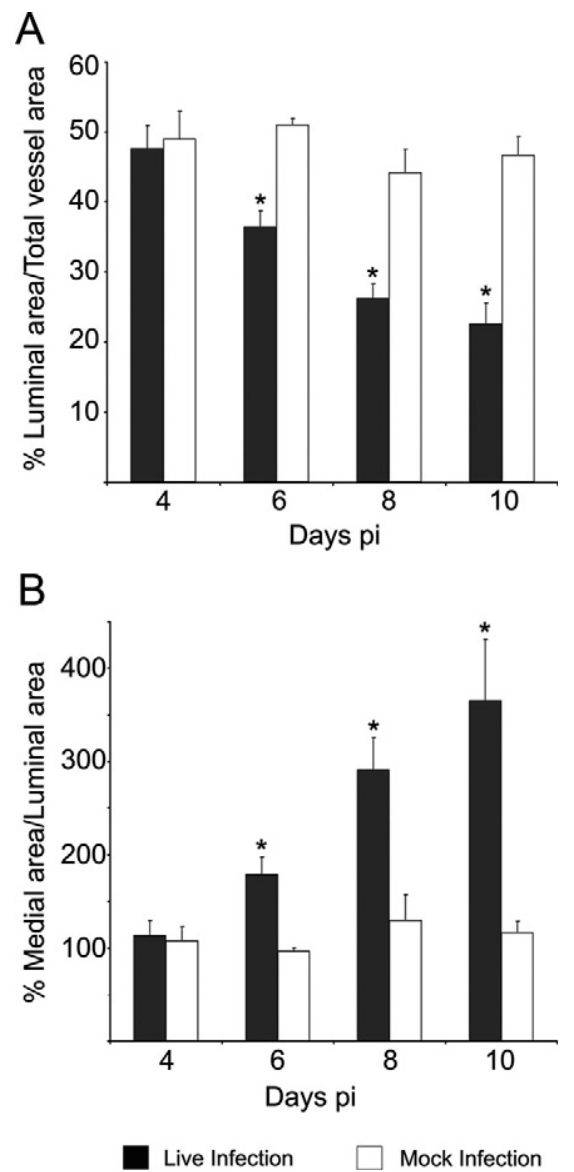

Figure 6. Quantitative representation of arterial wall thickening during $C$. pneumoniae infection. Thickening was quantified and represented as ratios of lumen to total vessel area, (A) and media to luminal area (B) (mean \pm SEM). ${ }^{*} P<0.02$ versus day 4 pi.

ted. ${ }^{23,42-44}$ Consistent with these findings, in the present study we have shown that $C$. pneumoniae can infect the coronary artery in culture and this infection promotes a stimulation of cell proliferation and intimal thickening. C. pneumoniae can infect and persist through multiple life cycles (which is typically 48 to 72 hours) ${ }^{2,27}$ in a coronary artery. At day 2 pi, C. pneumoniae IB distribution was found mostly in the endothelial layer (Figure 2A), which is expected because only the endothelium was exposed to the pathogen during the initial infection. On subsequent days, the C. pneumoniae IBs distribution was shifted and it was found almost exclusively in the smooth muscle cell media (Figure 2, B-E). Our data are consistent with previously published findings ${ }^{6,27,45}$ that the smooth muscle cell media is the preferred location of the $C$. pneumoniae propagation. C. pneumoniae infection did not induce endothelial cell apoptosis (data not shown), therefore the inability of the endothelial layer to support stable $C$. pneumoniae replication is probably attributable to the initial damage of the endothelial cells caused by the infection. ${ }^{27}$

Increasing levels of chlamydial HSP60 in the infected coronary segments (Figure 3B) further supported the assertion that $C$. pneumoniae was metabolically active in the coronary segments. Chlamydial HSP60 is synthe- 
sized only by metabolically active $C$. pneumoniae reticulate bodies and not the progeny C. pneumoniae elementary bodies, ${ }^{46}$ and a significant amount of chlamydial HSP6O is released into the circulation during infection. ${ }^{47,48}$ As such, the observed increase of chlamydial HSP60 protein $(\approx 2.5 \times)$ in the protein lysate of coronary segments may only represent a conservative estimate of the total C. pneumoniae in the infected vessels. According to these data, $C$. pneumoniae replicates and remains metabolically active in our novel ex vivo coronary artery model. Another important conclusion from these findings is that in vivo C. pneumoniae infection of the endothelial cells layer is sufficient to initiate propagation of the infection into the arterial media.

The infection of the isolated coronary artery with $C$. pneumoniae had structural implications for the arterial wall. The induction of PCNA would suggest that cell proliferation is stimulated in the artery after C. pneumoniae infection. Consistent with our previous work, ${ }^{27}$ the mechanism by which infection with C. pneumoniae augments the proliferation of VSMCs may involve stimulating the expression of endogenous HSP60. In the present study, the expression of h-HSP60 was increased in the tissue during C. pneumoniae infection (Figure 4A). Increasing PCNA expression confirmed the proliferative nature in the infected vessel. However, it remains uncertain from in vitro cell culture studies whether the proliferative responses ultimately result in structural thickening. Our data here now clearly show that $C$. pneumoniae infection can directly induce thickening of the arterial wall in the intimal region in as little as a few days after exposure. Overall, we have observed a significant increase in thickening of the media starting at day 6 pi. Also, we observed that a majority of infected vessels demonstrated a collapse in the circular geometry, most notably from day 8 and onward. We did not collect vessels beyond day 10 because most infected vessels were so severely affected that they exhibited a completely collapsed lumen beyond that time point. Such narrowing would be expected to be associated with clinically significant effects. Our data are in agreement with observations that $C$. pneumoniae seropositivity is associated with arterial thickening, ${ }^{49}$ and that infection by C. pneumoniae without other atherosclerotic factors can induce atherosclerotic events. ${ }^{50}$

One of the most important findings from this study is the demonstration that an active C. pneumoniae infection alone can lead to arterial thickening in the absence of host immune responses. Previous in vivo studies have not been able to distinguish the contributions of the host immune responses at the infected location from the direct molecular consequences of bacterial replication in the infected media. Our study was designed to ensure that the coronary arteries were isolated from host immune responses (including macrophages, T-cells) and other biological molecules (such as elevated cholesterol) that may contribute to lesion formation. The use of heat-inactivated C. pneumoniae as a control is appropriate to reduce the potential contribution to the thickening effect that occurs as a result of bacterial LPS or mechanical stress induced by our handling. Further, comparisons were undertaken using paired coronary segments from the same heart (Figure 5) to strengthen the reliability of our observations. Thus, our h-HSP60 and PCNA expression data suggest $C$. pneumoniae infection can directly lead to the proliferation of the smooth muscle cells within the intima strongly enough to ultimately produce arterial thickening, without contribution from the host immune system. Despite the absence of an active immune response, however, immune mediators such as proinflammatory cytokines and chemokines could be involved in this proliferative response. C. pneumoniae infection of vascular smooth muscle cells has been shown to stimulate production and release of interleukin (IL) $-6^{51}$ and monocyte chemoattractant protein (MCP) $-1,52$ two mediators known to have proliferative effects on vascular smooth muscle cells. ${ }^{53,54,55}$ Thus, both IL-6 and MCP-1 through autocrine or paracrine actions may additionally stimulate the Cpn effects beyond that which was observed in the present set of experiments. The addition of monocytes to the system could further enhance this effect, as coculturing of monocytes and vascular smooth muscle cells alone cannot only enhance the growth of $C$. pneumoniae ${ }^{56}$ but also stimulate the production of $\mathrm{IL}-6$ and MCP-1. ${ }^{57}$ These data do not dispute the importance of a host immune response as an essential contributor to the overall atherosclerotic plaque development process. Rather, the present work has provided important evidence that $C$. pneumoniae on its own can induce a significant component of atherosclerosis (cell proliferation and intimal thickening).

In conclusion, our data has provided the first direct evidence that an active C. pneumoniae infection alone, without contributions from a host immune system, can stimulate arterial thickening. This effect is associated with the up-regulation of h-HSP60 and PCNA expression. Our work has also demonstrated the feasibility of studying the molecular mechanisms of infection-induced atherosclerosis using an ex vivo coronary culture system.

\section{References}

1. Kuo C-C, Jackson LA, Campbell LA, Grayston JT: Chlamydia pneumoniae (TWAR). Clin Microbiol Rev 1995, 8:451-461

2. Moulder JW: Interaction of chlamydiae and host cells in vitro. Microbiol Rev 1991, 55:143-190

3. Hatch TP: Utilization of L-cell nucleoside triphosphates by Chlamydia psittaci for ribonucleic acid synthesis. J Bacteriol 1975, 122:393-400

4. Ceballos MM, Hatch TP: Use of HeLa cell guanine nucleotides by Chlamydia psittaci. Infect Immun 1979, 25:98-102

5. Quinn TC, Gaydos CA: In vitro infection and pathogenesis of Chlamydia pneumoniae in endovascular cells. Am Heart J 1999, 138:S507-S511

6. Hirono S, Pierce GN: Dissemination of Chlamydia pneumoniae to the vessel wall in atherosclerosis. Mol Cell Biochem 2003, 246:91-95

7. Yang ZP, Kuo CC, Grayston JT: Systemic dissemination of Chlamydia pneumoniae following intranasal inoculation in mice. J Infect Dis 1995, 171:736-738

8. Saikku P, Leinonen M, Mattila K, Ekman MR, Nieminen MS, Mäkelä $\mathrm{PH}$, Huttunen JK, Valtonen V: Serological evidence of an association of a novel Chlamydia, TWAR, with chronic coronary heart disease and acute myocardial infarction. Lancet 1988, 2:983-986

9. Thom DH, Wang SP, Grayston JT, Siscovick DS, Stewart DK, Kronmal RA, Weiss NS: Chlamydia pneumoniae strain TWAR antibody and 
angiographically demonstrated coronary artery disease. Arterioscler Thromb 1991, 11:547-551

10. Muhlestein JB, Hammond EH, Carlquist JF, Radicke E, Thomson MJ, Karagounis LA, Woods ML, Anderson JL: Increased incidence of Chlamydia species within the coronary arteries of patients with symptomatic atherosclerotic versus other forms of cardiovascular disease. J Am Coll Cardiol 1996, 27:1555-1561

11. Sander D, Winbeck K, Klingelhöfer J, Etgen T, Conrad B: Enhanced progression of early carotid atherosclerosis is related to Chlamydia pneumoniae (Taiwan acute respiratory) seropositivity. Circulation 2001, 103:1390-1395

12. Kuo C-C, Grayston JT, Campbell LA, Goo YA, Wissler RW, Benditt EP Chlamydia pneumoniae (TWAR) in coronary arteries of young adults (15-34 years old). Proc Natl Acad Sci USA 1995, 92:6911-6914

13. Yamashita K, Ouchi K, Shirai M, Gondo T, Nakazawa T, Ito H: Distribution of Chlamydia pneumoniae infection in the atherosclerotic carotid artery. Stroke 1998, 29:773-778

14. Mosorin M, Surcel HM, Laurila A, Lehtinen M, Karttunen R, Juvonen J, Paavonen J, Morrison RP, Saikku P, Juvonen T: Detection of Chlamydia pneumoniae-reactive $T$ lymphocytes in human atherosclerotic plaques of carotid artery. Arterioscler Thromb Vasc Biol 2000, 20:1061-1067

15. Kuo CC, Gown AM, Benditt EP, Grayston JT: Detection of Chlamydia pneumoniae in aortic lesions of atherosclerosis by immunocytochemical stain. Arterioscler Thromb 1993, 13:1501-1504

16. Maass M, Bartels C, Engel PM, Mamat U, Sievers HH: Endovascular presence of viable Chlamydia pneumoniae is a common phenomenon in coronary artery disease. J Am Coll Cardiol 1998, 31:827-832

17. Jackson LA, Campbell LA, Schmidt RA, Kuo CC, Cappuccio AL, Lee MJ, Grayston JT: Specificity of detection of Chlamydia pneumoniae in cardiovascular atheroma: evaluation of the innocent bystander hypothesis. Am J Pathol 1997, 150:1785-1790

18. Gurfinkel E, Bozovich G, Daroca A, Beck E, Mautner B: Randomised trial of roxithromycin in non-Q-wave coronary syndromes: rOXIS Pilot Study. ROXIS Study Group. Lancet 1997, 350:404-407

19. Sinisalo J, Mattila K, Valtonen V, Anttonen O, Juvonen J, Melin J, Vuorinen-Markkola $\mathrm{H}$, Nieminen MS: Effect of 3 months of antimicrobial treatment with clarithromycin in acute non-q-wave coronary syndrome. Circulation 2002, 105:1555-1560

20. Wiesli P, Czerwenka W, Meniconi A, Maly FE, Hoffmann U, Vetter W, Schulthess G: Roxithromycin treatment prevents progression of peripheral arterial occlusive disease in Chlamydia pneumoniae seropositive men: a randomized, double-blind, placebo-controlled trial Circulation 2002, 105:2646-2652

21. Hu H, Pierce GN, Zhong G: The atherogenic effects of chlamydia are dependent on serum cholesterol and specific to Chlamydia pneumoniae. J Clin Invest 1999, 103:747-753

22. Cao F, Castrillo A, Tontonoz P, Re F, Byrne Gl: Chlamydia pneumoniae-induced macrophage foam cell formation is mediated by Toll-like receptor 2. Infect Immun 2007, 75:753-759

23. Byrne Gl, Kalayoglu MV: Chlamydia pneumoniae and atherosclerosis: links to the disease process. Am Heart J 1999, 138:S488-S490

24. Kalayoglu MV, Byrne GI: Induction of macrophage foam cell formation by Chlamydia pneumoniae. J Infect Dis 1998, 177:725-729

25. Coombes BK. Mahony JB. Chlamydia pneumoniae infection of human endothelial cells induces proliferation of smooth muscle cells via an endothelial cell-derived soluble factor (s). Infect Immun 1999, 67:2909-2915

26. Coombes BK, Chiu B, Fong IW, Mahony JB: Chlamydia pneumoniae infection of endothelial cells induces transcriptional activation of platelet-derived growth factor-B: a potential link to intimal thickening in a rabbit model of atherosclerosis. J Infect Dis 2002, 185:1621-1630

27. Hirono S, Dibrov E, Hurtado C, Kostenuk A, Ducas R, Pierce GN Chlamydia pneumoniae stimulates proliferation of vascular smooth muscle cells through induction of endogenous heat shock protein 60 Circ Res 2003, 93:710-716

28. Wick G, Perschinka H, Millonig G: Atherosclerosis as an autoimmune disease: an update. TRENDS in Immunology 2001, 22:665-669

29. Huittinen $T$, Leinonen $M$, Tenkanen L, Manttari M, Virkkunen H, Pitkanen T, Wahlstrom E, Palosuo T, Manninen V, Saikku P: Autoimmunity to human heat shock protein 60 , chlamydia pneumoniae infection, and inflammation in predicting coronary risk. Arterioscler Thromb Vasc Biol 2002, 22:431-437

30. Wallin RPA, Lundqvist A, More, von Bonin A, Kiessling R, Ljunggren
$\mathrm{H}-\mathrm{G}$. Heat-shock proteins as activators of the innate immune system. TRENDS in Immunology 2002, 23:665-669

31. Ross R: Atherosclerosis- An inflammatory disease. N Engl J Med 1999, 340:115-126

32. Hsich E, Zhou YF, Paigen B, Johnson TM, Burnett MS, Epstein SE: Cytomegalovirus infection increases development of atherosclerosis in Apolipoprotein-E knockout mice. Atherosclerosis 2001, 156:23-28

33. Li L, Messas E, Batista EL Jr, Levine RA, Amar S: Porphyromonas gingivalis infection accelerates the progression of atherosclerosis in a heterozygous apolipoprotein E-deficient murine model. Circulation 2002, 105:861-867

34. Kol A, Sukhova GK, Lichtman AH, Libby P: Chlamydial heat shock protein 60 localizes in human atheroma and regulates macrophage tumor necrosis factor-alpha and matrix metalloproteinase expression. Circulation 1998, 98:300-307

35. Kol A, Bourcier T, Lichtman AH, Libby P: Chlamydial and human heat shock protein 60s activate human vascular endothelium, smooth muscle cells, and macrophages. J Clin Invest 1999, 103:571-577

36. Schett G, Xu Q, Amberger A, Van der Zee R, Recheis H, Willeit J, Wick G: Autoantibodies against heat shock protein 60 mediate endothelial cytotoxicity. J Clin Invest 1995, 96:2569-2577

37. Chen W, Syldath U, Bellmann K, Burkart V, Kolb H: Human 60-kDa heat-shock protein: a danger signal to the innate immune system. J Immunol 1999, 162:3212-3219

38. Kuo CC, Grayston JT: A sensitive cell line, HL cells, for isolation and propagation of Chlamydia pneumoniae strain TWAR. J Infect Dis 1990, 162:755-758

39. Furness G, Graham DM, Reeve P: The titration of trachoma and inclusion blennorrhoea viruses in cell cultures. J Gen Microbiol 1960 23:613-619

40. Saward L, Zahradka P: Coronary artery smooth muscle in culture: migration of heterogeneous cell populations from vessel wall. Mol Cell Biochem 1997, 176:53-59

41. Zettler ME, Prociuk MA, Austria JM, Massaeli H, Zhong G, Pierce GN OxLDL stimulates cell proliferation through a general induction of cell cycle proteins. Am J Physiol Heart Circ Physiol 2003, 284:H644-H653

42. Campbell LA, Kuo CC, Grayston JT: Chlamydia pneumoniae and cardiovascular disease. Emerg Infect Dis 1998, 4:571-579

43. Gupta S: Chlamydia pneumoniae, monocyte activation, and azithromycin in coronary heart disease. Am Heart J 1999, 138:S539-S541

44. Ouchi K: Chlamydia pneumoniae and atherosclerosis. Jpn J Infect Dis 1999, 52:223-227

45. Shor A, Kuo CC, Patton DL: Detection of Chlamydia pneumoniae in coronary arterial fatty streaks and atheromatous plaques. S Afr Med J 1992, 82:158-161

46. Marino J, Stoeckli I, Walch M, Latinovic-Golic S, Sundstroem H, Groscurth P, Ziegler U, Dumrese C: Chlamydophila pneumoniae derived from inclusions late in the infectious cycle induce aponecrosis in human aortic endothelial cells. BMC Microbiology 2008, 8:1-14

47. Heltai K, Kis Z, Burian K, Endresz V, Veres A, Ludwig E, Gönczöl E, Valyi-Nagy I: Elevated antibody levels against Chlamydia pneumoniae, human HSP60 and mycobacterial HSP65 are independent risk factors in myocardial infarction and ischaemic heart disease. Atherosclerosis 2004, 173:339-346

48. Andrié R, Braun P, Welsch U, Straube E, Höpp HW, Erdmann E, Lüderitz B, Bauriedel G: [Chlamydial and human heat shock protein 60 homologues in acute coronary syndromes. (Auto-)immune reactions as a link between infection and atherosclerosis]. Z Kardiol 2003, 92:455-465

49. Schmidt C, Hulthe J, Wikstrand J, Gnarpe H, Gnarpe J, Agewall S, Fagerberg B: Chlamydia pneumoniae seropositivity is associated with carotid artery intima-media thickness. Stroke 2000, 31:1526-1531

50. Fong IW, Chiu B, Viira E, Jang D, Mahony JB: De Novo induction of atherosclerosis by Chlamydia pneumoniae in a rabbit model. Infect Immun 1999, 67:6048-6055

51. Rodel J, Woytas M, Groh A, Schmidt K-H, Hartmann M, Lehmann M, Straube E: Production of basic fibroblast growth factor and interleukin- 6 by human smooth muscle cells following infection with Chlamydia pneumoniae. Infect Immun 2000, 68:3635-3641

52. Yang X, Coriolan D, Schultz K, Golenbock D, Beasly D: Toll-like receptor 2 mediates persistent chemokine release by Chlamydia pneumoniaeinfected vascular smooth muscle cells. Arterioscler Thromb Vasc Biol 2005, 25:2308-2314 
53. Ikeda U, Ikeda M, Oohara T, Oguchi A, Kamitani T, Tsuruya $Y$, Kano S: Interleukin 6 stimulateds growth of vascular smooth muscle cells in a PDGF-dependent manner. Am J Physiol 1991, 260:H1713-H1717

54. Viedt C, Vogel J, Athanasiou T, Shen W, Orth SR, Kubler W, Kreuzer $\mathrm{J}$ : Monocyte chemoattractant protein-1 induces proliferation and interleukin-6 production in human smooth muscle cells by differential activation of nuclear factor-kB and activator protein-1. Arterioscler Thromb Vasc Biol 2002, 22:914-920

55. Selzman C, Miller S, Zimmerman M, Gamboni-Robertson F, Harken A, Banerjee A: Monocyte chemotactic protein-1 directly induces human vascular smooth muscle proliferation. Am J Physiol Heart Circ Physiol 2002, 283:H1455-H1461

56. Puolakkainen M, Campbell LA, Lin T-M, Richards T, Patton D, Kuo C-C. Cell-to-cell contact of human monocytes with infected arterial smooth muscle cells enhances growth of Chlamydia pneumoniae. $\mathrm{J}$ Infect Dis 2003, 187:435-40

57. Chen L, Frister A, Wang S, Ludwig A, Behr H, Pippig S, Li B, Simm A, Hofmann B, Pilowski C, Koch S, Buerke M, Rose-John S, Werdan K, Loppnow $\mathrm{H}$ : Interaction of vascular smooth muscle cells and monocytes by soluble factors synergistically enhances IL-6 and MCP-1 production. Am J Physiol Heart Circ Physiol 2009, 296:H987-H996 